\title{
Surgery of common paralytic conditions
}

\author{
FRANK DUERKSON \\ Health Sciences Centre, 700 William Avenue, Winnipeg, Manitoba, \\ Canada R3E $0 Z 3$
}

Involvement and loss of function of nerves, cutaneous as well as trunk nerves, is the main cause of impairment. The subsequent disability leads to a handicap in leprosy.

Early intervention at the stage of impairment is the best way to prevent deformity, disability, the stigma and fear of leprosy. The next best is intervention at the stage of early deformity and disability before it becomes established and irreversible.

Early surgery can correct deformity and disability; the earlier it is done the better is the restoration of form and function.

A positive attitude and motivation on the part of the patient, doctor and all involved in this work, is essential.

The surgical team consists of a willing patient, a specially trained surgeon, trained physical and occupational therapists, a social worker and the operation theatre staff. Trunk nerve decompression can often help in restoring the function of a damaged nerve, especially when done early.

\section{Nerve decompression surgery}

The different procedures are:

1. External decompression, e.g. carpal tunnel release.

2. Epicondylectomy.

3. Anterior transposition.

4. Epineurotomy.

5. Internal decompression or neurolysis.

Successful surgery in leprosy is a result of teamwork, not only during but also before and after surgery, including at periodic follow-up visits for at least a year after surgery. 


\section{Nerve surgery}

Surgical decompression of a trunk nerve is indicated when medical treatment for nerve damage has failed or there is a nerve abscess. If after 4-6 weeks of corticosteroid treatment the neurological evaluation and symptoms do not show improvement, then surgical release of the nerve is indicated.

\section{METHODS}

In most cases, external release of compressing factors is adequate. In instances where the nerve is swollen with a thick epineurium, incision of the nerve sheath should be done. This needs an experienced surgeon and magnification with a loop during surgery. In case of a nerve abscess, careful removal of all infected necrotic tissues is required.

\section{Ulnar nerve}

This is the most common nerve of ten requiring surgical decompression to prevent trauma by compression, stretch and friction.

SURGICAL DECOMPRESSION OF THE ULNAR NERVE AT ELBOW

The ulnar nerve just above the elbow is commonly involved. It is thickened, sometimes nodular, with pain on movement of elbow, tenderness and a varying degree of sensory and motor paralysis. Sometimes there is severe tenderness; warmth and swelling indicating nerve abscess (Figure 1).

Occasionally the nerve has no pain or tenderness, but there is reduced nerve function; this is labelled as a quiet nerve paralysis. The role of surgery here is not yet established. The various techniques of surgery are:

1. Release of the roof of the fibro-osseous tunnel, the epitrochleo-olecranon canal. This is sufficient in most cases. The roof is formed of fibrous bands and the arching origin of flexor carpi ulnaris muscle. This has to be incised.

2. Some surgeons advocate other procedures like anterior transposition (subcutaneous or under the muscles) or epicondylectomy in order to eliminate traction injury. No conclusive evidence is available at present that any of these techniques are better than a simple release of external compressing factors.

3. Epineurotomy is release of the compressing thickened nerve sheath. This is indicated when the nerve sheath is much thickened and opaque. This surgery is delicate and needs experience and magnification during surgery.

\section{Median nerve}

The median nerve is compressed at the wrist in the carpal tunnel. A standard carpal tunnel release is indicated. Sometimes an epineurotomy is also required, as in the case of the ulnar nerve. The results of early surgery are good. 


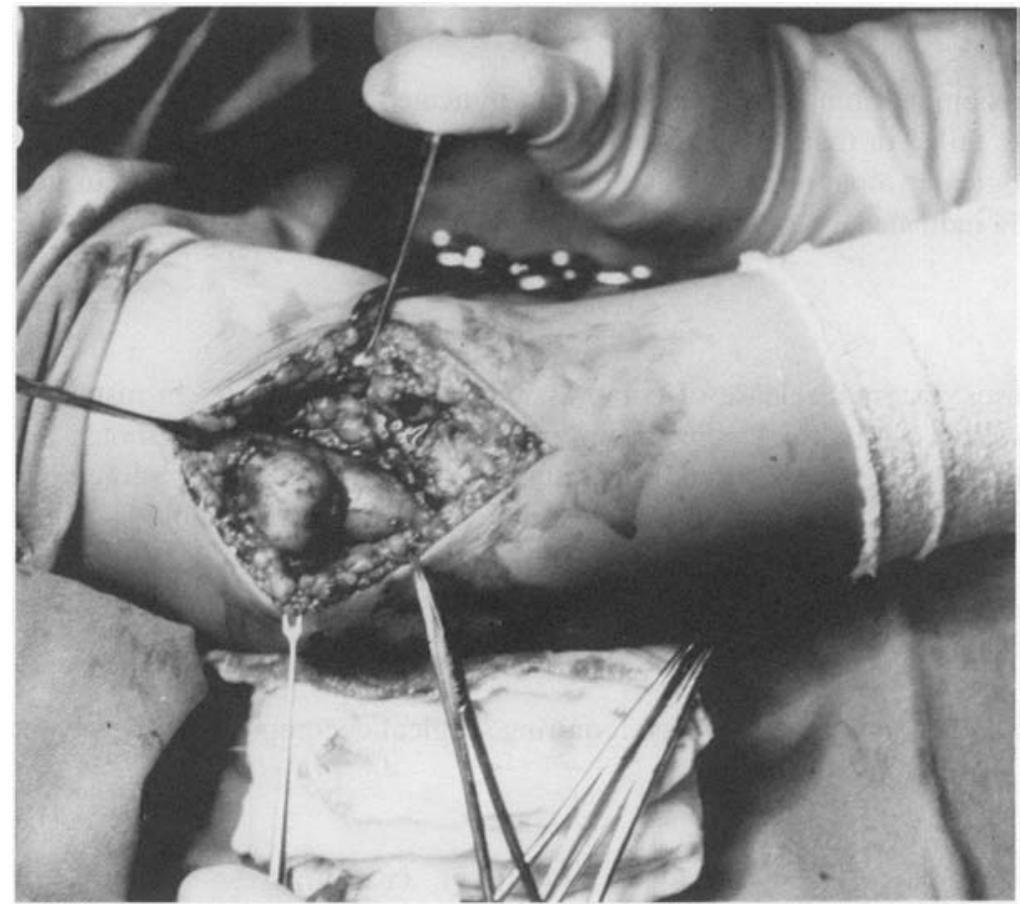

Figure 1. Ulnar nerve abscess.

\section{Posterior tibial nerve}

The posterior tibial nerve is compressed behind the ankle, in the tarsal tunnel. This is probably the most important nerve to release since permanent damage here leads to paralysis of the sensory supply of the whole of the sole of foot and paralysis of intrinsic muscles. At this site, the nerve and blood vessels travel together within a common neurovascular sheath. The artery often provides the main blood supply of the foot. Because of involvement and swelling of the nerve, the artery is secondarily compressed reducing the vascular supply of the foot. Early decompression releases from pressure both the artery and the nerve. The method of release is incision of the flexor retinaculam, of the neurovascular sheath, of the constricting calcaneal bands and sometimes epineurotomy. More work is required to establish this benefit.

\section{The common peroneal (lateral popliteal) nerve}

The common peroneal nerve is less frequently involved and responds better to conservative treatment with rest, splinting and steroids.

Occasionally decompression of this nerve is required, especially in case of a nerve abscess. The procedure is release of the fibrous bands overlying the nerve at the level of the neck of the fibula and release of the fibrous arch in the peroneal muscles through which the nerve passes. 
In leprosy, absence of controls in the study of nerve surgery has deprived it of an established place in prevention of deformity and disability. Individual experiences are, however, quite encouraging.

\section{Surgical treatment of deformities and disabilities secondary to permanent nerve damage}

This section describes the commonly performed reconstructive surgery procedures for restoration of form and function of the hand and foot in leprosy. Early diagnosis and treatment is the best prevention, next best is early treatment of nerve damage aimed at restoration of nerve function. When there is established paralysis and resultant deformity, early reconstructive surgery is desirable.

For most such deformities of hands and feet, very successful surgical solutions are available. The success depends on teamwork involving an experienced surgeon, physiotherapists and well-motivated patients. Care of the part with loss of sensation is of course essential to maintain the form and function restored by surgery.

RECONSTRUCTIVE SURGERY OF HAND

Tendon transfers can correct or improve most problems caused by paralysis of the hands and feet in leprosy.

Co-operation from an experienced physiotherapist and the availability of an experienced surgeon, who knows how to handle tissues gently and understands the biomechanics of tendon transfer surgery, is essential.

The hand has to be made fully mobile before surgery by methods of physical therapy including exercises, wax therapy correction of any contractures, correction of any adaptive shortening of the long flexors, erasing of trick movements and other measures.

Next comes training of the muscle-tendon complex to be transferred and explanation to the patient of the maximum restoration of form and function that this surgery is aiming at in his case and the nature of the co-operation required.

Protection of the transfer during the first 3 months by avoiding excessive stress and strain and repeated reinforcement of integration of the transfer at follow-up visits is essential to achieve a good result. The patient has to be encouraged to use the operated hand, but with care.

\section{Surgery for ulnar nerve paralysis (Figure 2)}

Clawing of the hand can easily be corrected by a variety of surgical techniques. Surgery is delicate and very exact, and must be accompanied by the patient learning techniques of care of the parts with loss of sensation since surgery only corrects the motor component. 

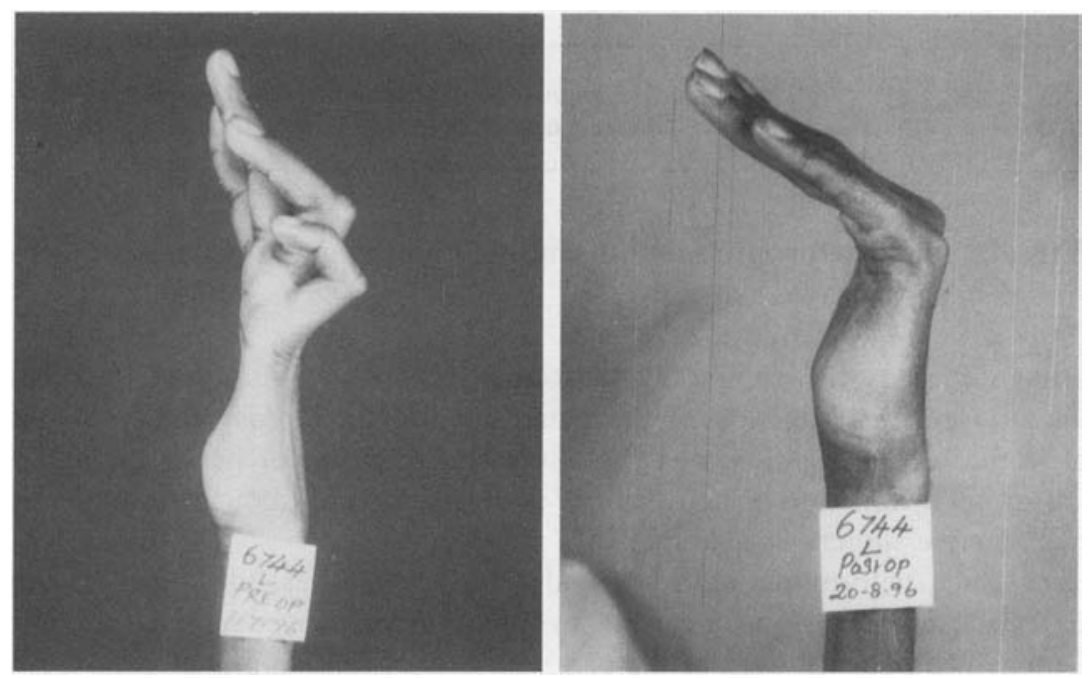

Figure 2. Ulnar paralysis repair. Four-tailed transfer of extensor carpi radialis longus into fibrous flexor pulleys. The tendon of flexor superficialis of one finger (usually the ring) or the palmaris longus is looped around all flexors, tunnelled to the radial side of the hand and inserted in the tendon of abductor brevis or flexor brevis of the thumb to restore pinch power.

\section{PROCEDURES}

The principle is correction of the muscular imbalance at the level of the metacarpophalangeal (MP) joints of the fingers and thumb. The common techniques are:

1. The transfer described by Dr Brand using the extensor carpi radialis longus as a motor extended by a graft, tendon or fascia lata. The insertion is into the lateral bands of the extensor expansion of the fingers in the proximal phalanx. The tendon is routed in front of the wrist and MP joints.

2. Transfer of flexor superficialis of the long or middle finger (first described by Bunnel) to the extensor expansion as above.

3. Zancolli's 'lasso' operation principle where the transfer, ECRL of flexor superficialis is inserted into the A1 (and part of A2) fibrous flexor pulley. This technique is specially useful in hypermobile fingers, where other techniques could cause a swan neck deformity.

\section{Surgery for median nerve paralysis (Figure 3)}

The disability involves loss of opposition and instability of the MP joint of the thumb.

The aim of surgery is restoration of stability of the MP joint and restoration of a three-finger pinch grasp.

\section{METHOD}

Replacement of opposition and of flexor brevis action by: (1) a single tendon transfer where the tendon is divided into two slips. Usually this tendon is the superficial flexor of the 


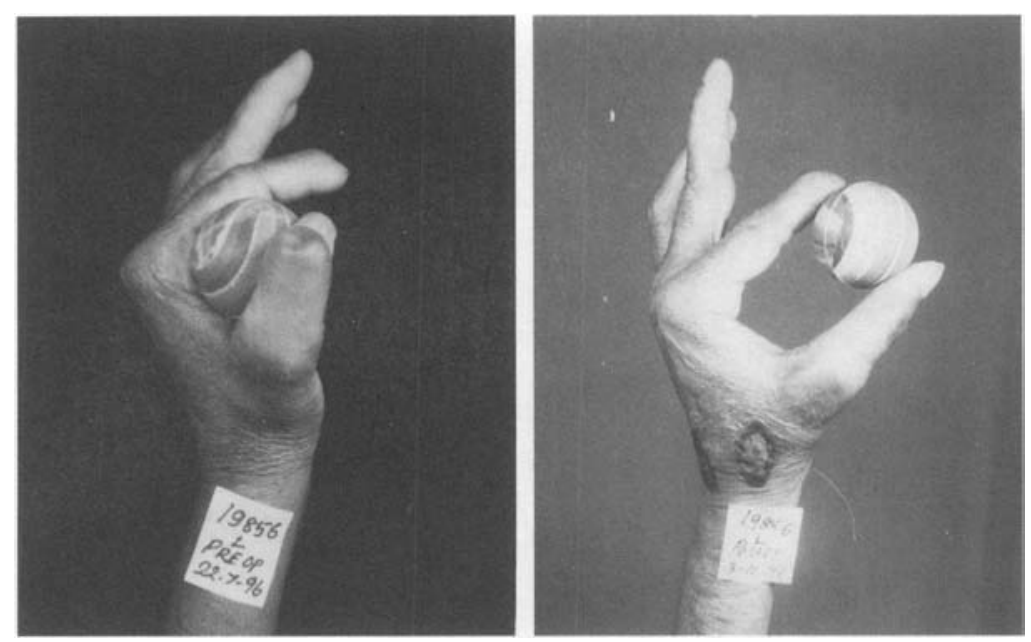

Figure 3. Surgery for median nerve paralysis.

ring finger as recommended by Dr Brand. Another popular transfer is using the extensor indicis proprious tendon routed around the ulnar border of the wrist to the thumb; (2) the second method is 'two-tendon transfer', also described by Dr Brand. One tendon restores abduction-oppositions and another the flexor brevis function.

For restoring the loss of opposition of the thumb, many procedures have been described. We routinely use the single tendon transfer described by Dr Brand. The flexor superficialis (sublimis) tendon of the ring finger is detached and then tunnelled from the ulnar side of the wrist to the thumb and divided into two slips. One goes around the neck of the thumb metacarpal and is sutured to the adductor pollicis tendon. This provides opposition. The other slip goes to the radial side of the thumb, volar to the axis of rotation of the MP joint and is inserted into the extensor pollicis longus tendon. This slip provides stability to the MP joint in flexion.

CORRECTION OF ATROPHY OF THE FIRST INTEROSSEOUS SPACE

The first web space becomes hollow because of wasting of the first interosseous muscle. This noticeable deformity is often a cause of stigma. Correction by a silicon implant or a dermal flat implant successfully corrects the deformity.

\section{Correction of foot-drop and clawed toes}

SURGERY OF COMMON PARALYTIC CONDITIONS OF THE FOOT IN LEPROSY

The two common conditions are foot-drop and clawed toes. Foot-drop can be partial or complete where all the dorsiflexors and peroneii are paralysed. Claw toes can be mobile or fixed with bony or soft tissue contractures.

Surgical correction prevents development of lateral border ulceration, rigid equinovarus foot and excessive pressure under the metatarsal heads leading to plantar ulcers. 

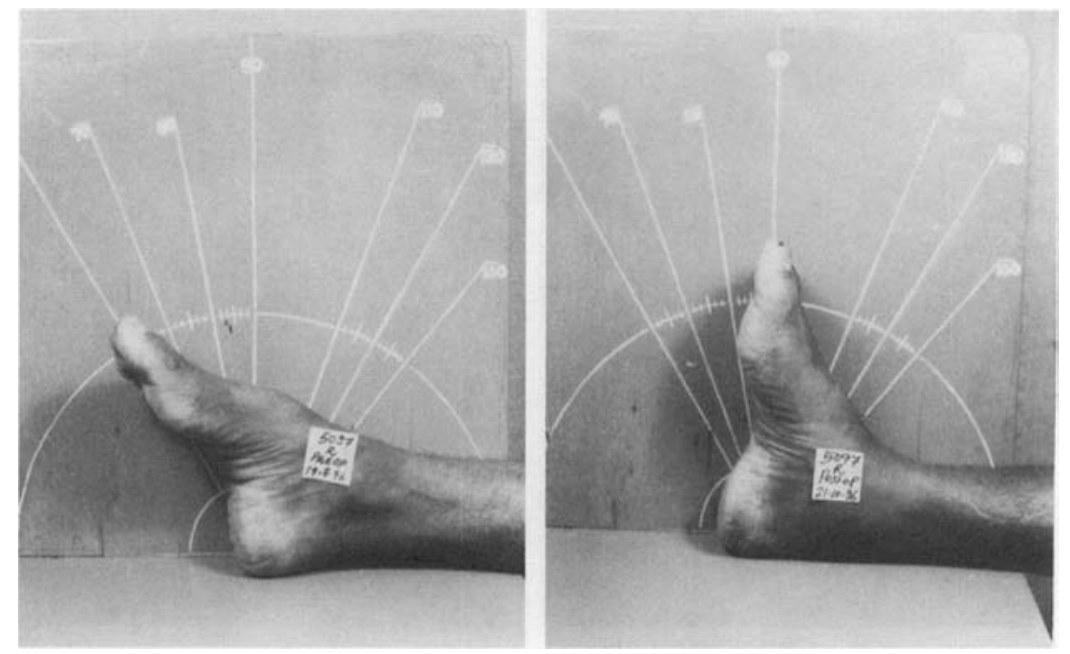

Figure 4. Correction of foot drop. Lengthening of the Achilles tendon, two-tailed tibialis posterior transfer and flexor to extensor transfer for claw toe.

\section{FOOT-DROP CORRECTION (FIGURE 4)}

The most common tendon transfer used is that of the tibialis posterior either through the interosseous membrane or subcutaneously around the medial aspect of tibia. Insertion is done either by two slips or by one slip. The two-slip insertion is done usually into the extensor hellucis longus and the extensor communis (of all lateral four toes) tendons. Insertion by single slip is often done either into bone or joint capsule in the middle of the foot.

Provision of appropriate footwear in maintaining a trouble-free foot cannot be overstressed.

\section{CLAWED TOES}

Clawing of toes often needs correction at the same time as correction of foot-drop. This is done, when the toes are mobile, by transfer of the toe flexors to the toe extensors thereby creating a new MTP flexor. When the clawing is fixed. it is corrected by joint fusion procedures.

Rigid equinovarus foot deformity is corrected by remodelling arthrodesis together with tibialis posterior transfer. 